\title{
Producto regional en Uruguay durante la Primera Globalización (1872-1908): desigualad decreciente y convergencia entre regiones
}

\author{
Pablo Castro Scavone* (DD \\ Instituto de Economía, Universidad de la República (Uruguay)

\section{Henry Willebald (DD} \\ Instituto de Economía, Universidad de la República (Uruguay)
}

\section{INFORMACIÓN DEL ARTÍCULO}

Historia del artículo:

Recibido: 22 de enero de 2020

Aceptado: 22 de marzo de 2021

On-line: 20 de octubre de 2021

\section{Códigos JEL:}

N5

N6

N9

R12

\section{Palabras clave:}

Desigualdad regional

Convergencia regional

Uruguay

\section{JEL classification:}

N5

N6

N9

R12

\section{Keywords:}

Regional inequality

Regional convergence

Uruguay

\begin{abstract}
R E S U M E N
Se presentan las primeras estimaciones del producto regional en Uruguay durante la Primera Globalización (desde la década de 1870 hasta los años previos a la Primera Guerra Mundial). Los resultados permiten identificar una tendencia descendente e irregular de la desigualad regional del producto per cápita consistente con un proceso de convergencia de ingresos entre departamentos. La irregularidad de la trayectoria permitiría corroborar, entonces, la actuación de fuerzas centrífugas y centrípetas alternado influencias durante el período. Las fuerzas que tendieron a desconcentrar la producción fueron la combinación de abundantes recursos naturales aptos para la producción ganadera en todo el territorio con la reducción de los costos de transporte que habría permitido volcar con fluidez la producción agropecuaria a Montevideo y, a través de su puerto, al mercado mundial. Las fuerzas centrípetas habrían respondido a un proceso caracterizado por la sostenida importancia de Montevideo como centro urbano y administrativo, mercado de bienes y servicios y centro dinámico del mercado laboral nacional. A ello se habría sumado la creciente importancia de la actividad comercial y financiera (y sus potencialidades para viabilizar el desarrollo industrial) en torno a Montevideo, la cual fue interrumpida por la crisis económica y financiera de 1890-1891. De hecho, esta actuó como una fuerza igualadora del período. El resultado fue abrir el siglo xx con niveles de desigualdad regional inferiores a los registrados en la década de 1870 .
\end{abstract}

@ 2021 Asociación Española de Historia Económica

\section{Regional value added in Uruguay during the First Globalization (1872-1908): decreasing inequality and convergence}

\begin{abstract}
A B S T R A C T
We present estimates of regional GDP in Uruguay during the First Globalization (from the 1870s to the years prior to the World War I). Our results show a decreasing and irregular trend in the regional inequality which is consistent with a process of income convergence between provinces. The irregularity of the trajectory would be evidence of the performance of centrifugal and centripetal forces that alternated influences during the period. The forces that tended to decentralize production were the combination of abundant natural resources suitable for livestock production throughout the territory and the reduction of transport costs that made easy access to Montevideo possible, as well as, through its port, to the global market. Centripetal forces would have responded to a process characterized by the increasing importance of Montevideo as an urban and administrative center, a huge market for goods and services and a dynamic centre of labour market. In addition, in Montevideo, commercial and financial activities (and their potential for making industrial development more flexible) were increasingly important, interrupted only by the economic and financial crisis of 1890-1891. In fact, the crisis constituted one of the main equalizing forces of the period. The result was that at the beginning of the 20th century levels of regional inequality were lower than those recorded in the 1870s.
\end{abstract}

(c) 2021 Asociación Española de Historia Económica

\footnotetext{
* Autor para correspondencia. Correo electrónico: pablo.castro@iecon.ccee.edu.uy (Pablo Castro Scavone). 


\section{Introducción}

En el análisis del desempeño económico de largo plazo, la caracterización territorial e histórica del desarrollo constituye una dimensión crecientemente presente. En Uruguay, este tipo de consideraciones solo ha sido abordado para las últimas décadas del siglo xx (Rodríguez Miranda, 2010a, 2010b), y se cuenta únicamente con información cuantitativa parcial desde los años de 1960 en adelante ${ }^{1}$. Los estudios han privilegiado la unidad administrativa - departamento - como entidad de análisis. La información refiere a las diecinueve unidades administrativas en las cuales está dividido Uruguay que, como bien refleja el mapa 1 , constituyen espacios de dimensión y condición diversa. La caracterización fronteriza de algunos departamentos con sus dos grandes vecinos -al noreste con Brasil, en el litoral oeste con Argentina, y la salida al mar de otros (al Río de la Plata (hacia el suroeste) y al océano Atlántico (hacia el sureste)- dan cuenta de ello.

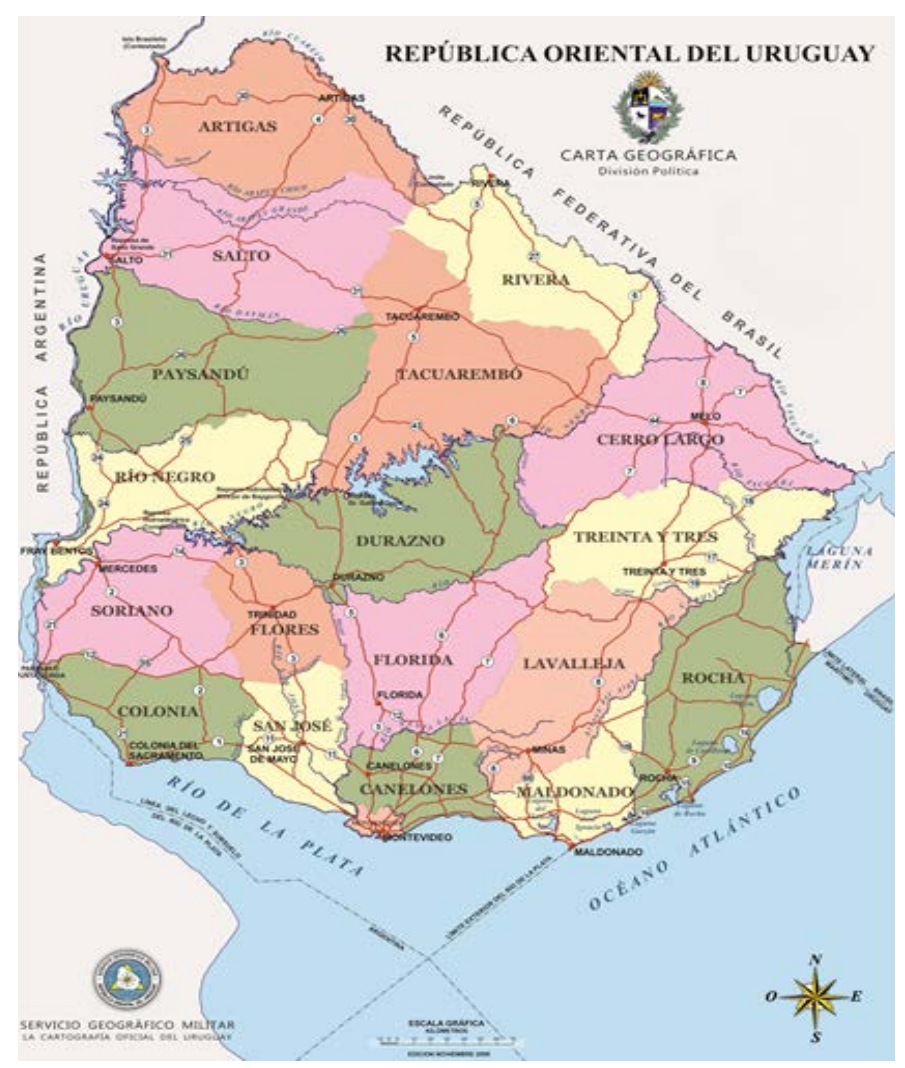

Mapa 1. Departamentos de Uruguay

Fuente: Servicio Geográfico Militar (http://www.sgm.gub.uy).

Entre 1962 y 1984 no hay estimaciones, y tampoco las hay para las décadas anteriores. Este trabajo contribuye con los esfuerzos recientes por llenar este vacío. Rodríguez Miranda y Goinheix (2018) presentan estimaciones del VAB departamental y sectorial para el período 1981-2008, en tanto que Martínez-Galarraga et al. (2019) dan cuenta de los criterios de esti-

\footnotetext{
1 BROU (1965), UDELAR (1995, 1998), OPP (2005a, 2005b, 2016a, 2016b), Molinari y Prieto (2011), Alonso Pérez y Risso (2012). Ver en Castro Scavone y Willebald (2019) un repaso de estos antecedentes.
}

mación de los VAB departamentales para 1908, 1936, 1955, 1961 y 1975 (once sectores). Ambos trabajos utilizan metodologías similares y permiten contar con una panorámica de un siglo de desigualdad regional, que ha quedado bien sintetizada en Martínez-Galarraga et al. (2020).

En el presente artículo se proponen estimaciones para el último tercio del siglo xIx hasta los años previos a la Primera Guerra Mundial - período identificado con la Primera Globalización- considerando, al menos, un año por década. La historiografía uruguaya coincide en destacar que el modelo agroexportador habría llegado a su agotamiento hacia los años de la Gran Guerra, en tanto que la década de 1920 constituiría un período de transición hacia un modelo diferente y basado en la industrialización sustitutiva (Millot y Bertino, 1996; Bertino et al., 2005). El último tercio del siglo xix hasta la primera década del xx constituye una unidad analítica en sí misma que justifica su abordaje particular. Fue un lapso de la historia económica de Uruguay caracterizado por una importante expansión del producto, creciente inserción exportadora -bienes primarios de origen agropecuario- $\mathrm{y}$ ritmos de aumento de la población que no volverán a repetirse en las décadas subsiguientes (con elevados índices de inmigración).

Este trabajo no se trata de un esfuerzo aislado, sino que en los últimos años el estudio de la desigualdad regional ha adquirido creciente interés en la historia económica. A partir del estudio pionero de Geary y Stark (2002) y la metodología sugerida por estos autores, así como el posterior de Crafts (2005), una serie de trabajos ha presentado nuevas estimaciones para países europeos: Buyst (2011) para Bélgica; Rosés et al. (2010) y Martínez-Galarraga et al. (2015) para España; Felice (2011) para Italia; Badia-Miró et al. (2012) para Portugal, y Enflo et al. (2014) para Suecia, entre otros.

El objetivo de este trabajo es el de ofrecer una contabilización del desempeño territorial histórico en Uruguay e identificar algunos hechos estilizados para establecer conjeturas y construir nuevas hipótesis de trabajo.

Los principales resultados son los siguientes. Es muy significativo el alto peso que históricamente ha tenido Montevideo en la generación local de valor agregado. Sin embargo, este rasgo no impidió la ocurrencia de un proceso de convergencia de ingresos per cápita entre regiones, lo cual no hizo más que confirmar la tendencia decreciente de la evolución de la desigualdad. De todos modos, la evolución igualadora no estuvo exenta de reversiones. Tanto en la década de 1880 como en la primera del siglo xx, las fuerzas centrípetas habrían tenido una mayor influencia en el aumento de los niveles de desigualdad. Esta larga evolución dio como resultado condiciones de localización de la producción que se expresaron en un patrón de ingresos per cápita con dos características principales. En primer lugar, el liderazgo de la capital se verificó, prácticamente, durante todo el período. En segundo lugar, esta persistencia contrasta con la transformación ocurrida en los restantes departamentos. Hacia la década de 1870 , el segundo núcleo de regiones ricas se constaba al norte del río Negro, donde la baja densidad poblacional se combinaba con abundantes recursos naturales. En cambio, hacia 1908 parece conformarse una zona relativamente más rica, que adopta una forma de L e incluye a los departamentos del sur y del litoral del país, siendo este un rasgo estructural de la distribución regional de la producción de Uruguay en el largo plazo.

El artículo se ordena de la siguiente manera. Luego de esta introducción, se presenta el marco conceptual y las principales 
hipótesis que guían esta investigación (sección 2). Se presenta la metodología de construcción de las series, se repasan las decisiones adoptadas para las actividades productivas (sección 3) y se exponen los resultados, identificándose algunos hechos estilizados (sección 4). Finalmente, se concluye (scción 5).

\section{Marco conceptual, hipótesis de trabajo y estrategia empírica}

La teoría de comercio neoclásica -modelo Heckscher-Ohlin- argumenta que los ingresos regionales difieren de acuerdo a las distintas dotaciones de factores y sus precios. El teorema de la igualación en el precio de los factores es optimista en cuanto a los efectos de la integración de mercados. El aumento del intercambio conduce a la igualación en el precio de los factores productivos (capital, trabajo) entre regiones ${ }^{2}$, pese a admitir la ocurrencia de diferentes especializaciones productivas. $\mathrm{Si}$, además de los precios, también se reducen los diferenciales de dotación entre regiones, disminuirían las disparidades regionales de ingresos. Esta es una argumentación que se identifica con lo que la literatura denomina los factores de primera generación. Esta conceptualización ha sido desafiada por la nueva geografía económica (new economic geography, $N E G$ ), la cual resulta menos optimista respecto a las consecuencias de la integración económica ${ }^{3}$.

Los modelos de la NEG se construyen en torno a la idea de que la diferenciación de productos, retornos crecientes a escala y reducción en los costos de transporte pueden generar externalidades pecuniarias en las elecciones de localización de firmas y trabajadores. Estos factores pueden llevar a procesos de aglomeración espacial y dispares especializaciones entre regiones. Los trabajadores tienden a concentrarse en una localización dada, razón por la cual el desplazamiento en la demanda aumenta el incentivo de las firmas a concentrar la producción en ciertos territorios. Además, los trabajadores pueden obtener un wage-premium en estos sitios dada la presencia de externalidades marshalianas y los subsiguientes mayores niveles de productividad. De este modo, la NEG argumenta la ocurrencia de concentraciones regionales aún en presencia de integración de mercados, lo cual se identifica con lo que la literatura denomina los factores de segunda generación.

En un trabajo clásico, Williamson (1965) argumenta que la desigualdad regional puede ser creciente en las primeras etapas del desarrollo económico moderno y descendente en etapas más avanzadas. En el largo plazo, la evolución simultánea de crecimiento, cambio estructural, integración económica e industrialización seguiría una evolución de la desigualdad regional con forma de U invertida. Varios autores -Caselli y Coleman (2001), la proponen para EE.UU.- destacan la relevancia del cambio estructural en la evolución de las desigualdades regionales y la relacionan con la desigual distribución territorial de la producción industrial.

A juzgar por estas aproximaciones, la expectativa de resultado que surge para este trabajo es que las fuerzas centrífugas debieron haber sido las dominantes y promovedoras, en consecuencia, de la desconcentración del ingreso y de un creciente

\footnotetext{
${ }^{2}$ El teorema requiere de una larga lista de supuestos (Samuelson, 1949; Leamer, 1995).

${ }^{3}$ Ver Baldwin et al. (2003) y Fujita et al. (1999).
}

acercamiento entre departamentos. En primer lugar, la dotación de tierra para la producción agropecuaria se distribuye -con relativa homogeneidad- en todo el territorio, hasta tal punto que la producción ganadera es posible desarrollarla en todas las tierras del país prácticamente sin excepciones ${ }^{4}$. Asimismo, la ausencia de cambio estructural durante la Primera Globalización (Román y Willebald, 2019) habría obrado restando presiones hacia la concentración, pues más allá de empujes esporádicos, la industrialización no fue dominante hasta bien avanzado el siglo xx. En segundo lugar, la reducción de los costos de transporte en el territorio - por la rápida expansión del ferrocarril-, que permitió volcar con fluidez la producción agropecuaria a Montevideo y, a través de su puerto, al mercado mundial, habría actuado como una fuerza integradora de mercados habilitando producciones rentables en muchos puntos del territorio, pero cuyo mercado principal no era el montevideano. La única fuerza que habría obrado claramente hacia la concentración de la actividad económica sería la creciente importancia de Montevideo como centro urbano y administrativo, mercado de bienes y servicios y polo dinámico del mercado laboral. La relación de ambos tipos de fuerza se expresa en la irregular trayectoria del período, aunque con el predominio de las fuerzas de primera generación y guiadas por una lógica à la Heckscher-Ohlin.

Esa evolución ocurrió en un escenario de sostenida integración de la economía uruguaya en el mercado internacional, con lo que quedaba expuesta a los movimientos de factores que caracterizaron el período. La relación entre integración económica internacional y evolución de la desigualdad regional merecería un estudio específico (para lo cual este período es particularmente atractivo). Para el alcance de este artículo, baste decir que se trató de un proceso que habría intensificado, y a veces dado lugar, a los movimientos antes señalados. La economía uruguaya se transformó en captadora neta de capital y trabajo ${ }^{5}$. El primero se expresó, fundamentalmente, en obras de infraestructura y transporte que habrían contribuido a la reducción de los costes de traslado de bienes (y personas) dentro del territorio, mientras que el factor productivo trabajo se concentró fundamentalmente en Montevideo, lo cual habría abaratado el coste de la mano y acortado las distancias prevalecientes al inicio del período. Estos factores, que habrían actuado como tendencias igualadoras, habrían sido parcialmente compensados por la acumulación de capital humano en la capital, producto de la llegada de contingentes poblacionales con conocimientos incorporados y no menores a los de la población residente (oficios, negocios, saberes). Buena parte del aumento del índice de Gini de la década de 1880 (como se verá) pudo haber incorporado este efecto.

Para testar aquellas hipótesis es necesario recorrer varias etapas analíticas. En primer lugar, se requiere la construcción de las series de datos. Para algunos años seleccionados se calcularon los VAB sectoriales departamentales y, a partir de su agregación, los productos por regiones, los cuales fueron expresados en términos per cápita a partir de las estimaciones de población. Luego, se identificaron varios hechos estilizados relacionados con: a) diversificación-especialización productiva; b) VAB per cápita; c) desigualdad regional, y d) convergencia/divergencia.

\footnotetext{
${ }^{4}$ Uruguay nunca fue durante el período un productor relevante de bienes agrícolas (como sí lo fue Argentina con los cereales).

${ }^{5}$ Ver Lezama y Willebald (2019) para una revisión de ambas evoluciones.
} 


\section{Metodología de construcción de las series}

Con el propósito de cubrir las últimas tres décadas del siglo xIx y la primera década del xx, se consideraron seis años de referencia (benchmarks temporales): 1872, 1884, 1890, 1895, 1900 y 1908. Al comienzo de ese período aún no estaba configurada la actual división administrativa compuesta por diecinueve departamentos. En 1880 se crearon Río Negro y Rocha a partir de territorios de Paysandú y Maldonado, respectivamente. Entre 1884 y 1885 fueron creados Artigas, Flores y Rivera a partir de territorios de Salto, San José y Tacuarembó, respectivamente (Peralta, 2008). A los efectos de comparar el desempeño departamental durante un período más extenso, se optó por utilizar la actual división administrativa como referencia. Esto implicó la necesidad de asignar producción a departamentos en todo el período de análisis, estimando la que les hubiera correspondido a departamentos que aún no habían sido creados, como Río Negro y Rocha en 1872 y Artigas, Flores y Rivera en 1872 y 1884 (ver anexo online) ${ }^{6}$.

La estimación del VAB departamental en el siglo xIx se enfrenta a importantes vacíos de información que requiere basarse en distintas variantes de la estimación indirecta. El desafío es la selección correcta de coeficientes (claves de distribución) consistentes a nivel sectorial y que permitan diferenciar niveles entre el departamento y el promedio de la economía, y así identificar estructuras departamentales ${ }^{7}$.

El VAB total y sectorial (minería, agricultura y pecuaria, industria manufacturera, construcción, comercio, suministro de electricidad, gas y agua, transporte y almacenamiento, comunicaciones, servicios de intermediación financiera, Administración pública, actividades inmobiliarias, empresariales y de alquiler, y otros servicios) está tomado de Román y Willebald (2019) (a precios corrientes). Con el objetivo de atenuar posibles efectos de años atípicos, se utilizaron promedios trianuales de VAB sectoriales.

Ante la inexistencia de una serie anual de población por departamentos para el período, fue necesario realizar algunas estimaciones. Se parte de la información reportada en los censos nacionales de población realizados en 1860 y 1908. Dado que en 1860 aún no estaba constituida la actual división administrativa, fue necesario realizar algunos supuestos (Castro Scavone y Willebald, 2019) para obtener la población de Artigas, Rocha, Flores, Río Negro y Rivera cuando estos, en 1860, aún no habían sido creados. Una vez que se contó con información de los diecinueve departamentos para 1860 y 1908, se realizaron interpolaciones y se obtuvieron las estructuras de población para los años de la investigación. Finalmente, se

\footnotetext{
${ }^{6}$ La alternativa hubiera sido trabajar con la división territorial vigente en 1872 ("unidad territorial mínima"). Sin embargo, condicionar toda la serie por solo dos puntos temporales parece inconducente. Más aun considerando el interés de esta investigación de abrir la posibilidad de compatibilizar estimaciones con las disponibles para el siglo xx y lograr una aproximación al tema de largo plazo. Si bien la opción que aquí se propone puede entrañar sus riesgos, se considera que los beneficios analíticos los compensan.

${ }^{7}$ Un método de estimación indirecta que produce resultados robustos es el propuesto en Geary y Stark (2002). Si bien los requerimientos de información estadística no son exagerados -datos de población activa (u ocupada) y salarios (por región y actividad)-, en la presente investigación se está lejos de contar con información suficiente de esas variables. Solo se la utiliza para la Administración pública y una versión alternativa para el sector agropecuario.
}

reescalaron los valores para hacerlos coincidir con la información agregada que se aporta en Nathan (2014) y Pellegrino (s. f.) para $1872,1884,1890,1895$ y 1900 . Ambos trabajos constituyen los esfuerzos más recientes de estimación8.

La estimación del VAB por departamentos se enfrenta a un grupo de problemas que debe ser abordado de manera conjunta. La información necesaria para obtener claves de distribución resulta, en muchos casos, discontinua en el tiempo, lo que imposibilita cubrir los benchmarks y obliga al uso de métodos de interpolación u, otras veces, resulta muy heterogénea, lo cual imposibilita el armado de estructuras departamentales completas ${ }^{9}$. A continuación se presentan las principales decisiones metodológicas y fuentes de información utilizadas; el apéndice online ofrece mayor detalle.

La estimación para el sector agropecuario siguió una metodología próxima a la de Geary y Stark (2002) al tomar en consideración la productividad física de la tierra -en lugar de la productividad laboral $-\mathrm{y}$, como referencia, el stock de ganado y la producción de cereales, uva y horticultura. Las fuentes fueron los censos ganaderos de 1860, 1900 y 1908 -con información adicional derivada de Acevedo (1933) - y anuarios estadísticos (AE) $(1884,1892,1895$ y 1900).

Para la minería se consideró una clave de distribución que combinó la producción de oro para Rivera (Baumann, 2017) y la distribución de la producción de canteras a partir de las patentes de giro ${ }^{10}$ obtenidas de $\mathrm{AE}(1890,1895,1900,1908)$.

La estimación para la construcción consideró la información proveniente de la Dirección General de Impuestos Indirectos en su capítulo "Riqueza pública», referida a la extensión de superficie edificada para los años 1885, 1890, 1895, 1900 y 1908. Los años anteriores se estimaron de acuerdo a la evolución de la población.

Para la Administración pública se utilizó la metodología de Geary y Stark (2002) a partir de la información que bridan los Presupuestos Generales de Gastos del Estado de 1871, 1884, 1890, 1893-1894, 1902-1903, Martínez Rodríguez (2020) y Sinisclachi (2019) sobre salarios y ocupados del sector público por departamento.

La importancia del sector de electricidad, gas y agua a nivel departamental fue deducida, únicamente, a partir de la generación de energía eléctrica (Bertoni, 2002).

Para los sectores de industria manufacturera, comercio, restaurantes y hoteles, servicios financieros y otros servicios se utilizó como clave de distribución la estructura derivada del pago de las patentes de giro, cuya información fue obtenida de Vaillant (1873), AE $(1884,1890,1895,1900,1908)$ y su valoración a partir del Libro de leyes y decretos del Uruguay (1884, 1890, 1895, 1900, 1908).

La estimación de transporte y almacenamiento tomó como referencia los kilómetros y la densidad de las principales vías de transporte (postas de diligencia, ferroviario y fluvial). La elaboración de las series se realizó a partir de Baracchini (1981) y $\operatorname{AE}(1884,1890,1895,1900,1908)$.

Para el sector de comunicaciones se siguió el mismo procedimiento que el anterior, pero considerando únicamente la

\footnotetext{
${ }^{8}$ Se agradece a la profesora Cabella por aconsejarnos en este punto.

${ }^{9}$ La información de los censos departamentales fue desestimada por esta razón.

${ }^{10}$ Se trata de un tipo de impuesto que "grava los beneficios de la industria, del comercio y de las profesiones liberales» (Acevedo, 1936, p. 605).
} 
densidad de vías férreas. Esta decisión se basó en que la red telegráfica es un buen indicador del desarrollo de las comunicaciones en el período analizado y que el tendido de la red siguió la pauta de instalación de estaciones ferroviarias (Barracchini, 1981).

Finalmente, la dinámica de las actividades inmobiliarias, empresariales y de alquiler se consideró determinada únicamente por las transacciones de bienes inmuebles. La cantidad de viviendas departamentales correspondiente a 1908 (Instituto Nacional de Estadística) fue estimada de acuerdo a la cantidad de población y valorizada según la renta media de la tierra para uso productivo (Balbis, 2005; Willebald, 2015).

\section{Resultados}

En esta sección se observan las características de la estructura productiva de los departamentos a partir de la construc- ción de indicadores de especialización y diversificación productiva, se analiza la distribución regional del ingreso y su evolución comparada (convergencia/divergencia).

\subsection{Especialización y diversificación productiva}

El primer resultado destacado es el liderazgo de la capital del país, Montevideo, durante todo el período de estudio. La mayor parte del VAB generado en la economía ( $48 \%$ en el promedio del período) fue montevideana, mientras que los restantes departamentos mostraron bajos ratios de participación (tabla 1$)^{11}$. Los departamentos con mayores participaciones se nuclearon en el sur y litoral del país, coincidiendo con las zonas de mejores tierras para la actividad agropecuaria (Millot y Bertino, 1996), una conectividad alta - por tierra y fluvial- y una histórica cercanía con Buenos Aires y las provincias del Litoral argentino.

\section{Tabla 1}

Estructura departamental del VAB (\%), 1872-1908

\begin{tabular}{|lrrrrrrr|}
\hline Departamento & $\mathbf{1 8 7 2}$ & $\mathbf{1 8 8 4}$ & $\mathbf{1 8 9 0}$ & $\mathbf{1 8 9 5}$ & $\mathbf{1 9 0 0}$ & $\mathbf{1 9 0 8}$ & Promedio 1872-1908 \\
\hline Montevideo & $\mathbf{4 6 , 5}$ & $\mathbf{4 5 , 9}$ & $\mathbf{5 4 , 9}$ & $\mathbf{4 8 , 0}$ & $\mathbf{4 5 , 8}$ & $\mathbf{4 8 , 6}$ & $\mathbf{4 8 , 3}$ \\
\hline Colonia & $\mathbf{4} 4$ & 5,7 & 5,3 & 5,3 & 8,1 & 6,2 & 5,8 \\
\hline Canelones & 2,9 & 4,0 & 3,4 & 4,3 & 6,0 & 6,7 & 4,6 \\
\hline Paysandú & 5,6 & 5,6 & 3,9 & 4,2 & 3,7 & 3,2 & 4,4 \\
\hline Salto & 4,7 & 4,2 & 3,4 & 3,9 & 3,7 & 3,5 & 3,9 \\
\hline Soriano & 3,7 & 3,5 & 2,9 & 3,4 & 3,3 & 3,1 & 3,3 \\
\hline Cerro.Largo & 3,4 & 3,5 & 2,9 & 3,2 & 2,6 & 2,7 & 3,0 \\
\hline Tacuarembó & 3,9 & 3,3 & 2,8 & 3,1 & 2,5 & 2,7 & 3,0 \\
\hline Durazno & 3,0 & 3,4 & 2,7 & 3,0 & 2,7 & 2,6 & 2,9 \\
\hline Florida & 2,5 & 2,6 & 2,2 & 2,8 & 2,8 & 2,8 & 2,6 \\
\hline Maldonado & 3,2 & 2,6 & 2,0 & 2,3 & 2,2 & 2,2 & 2,4 \\
\hline Artigas & 2,9 & 2,6 & 2,0 & 2,6 & 2,1 & 2,1 & 2,4 \\
\hline San.José & 2,0 & 2,0 & 1,7 & 2,2 & 3,2 & 2,7 & 2,3 \\
\hline Río.Negro & 2,1 & 2,6 & 2,3 & 2,7 & 2,2 & 1,8 & 2,3 \\
\hline Treinta.y.Tres & 2,1 & 2,2 & 1,8 & 2,1 & 1,8 & 1,8 & 2,0 \\
\hline Rocha & 2,2 & 1,8 & 1,7 & 2,1 & 2,0 & 1,9 & 2,0 \\
\hline Lavalleja & 1,6 & 1,3 & 1,2 & 1,3 & 2,2 & 2,2 & 1,6 \\
\hline Rivera & 1,7 & 1,5 & 1,4 & 1,7 & 1,4 & 1,9 & 1,6 \\
\hline Flores & 1,4 & 1,8 & 1,6 & 1,8 & 1,5 & 1,3 & 1,6 \\
\hline
\end{tabular}

Fuente: elaboración propia.

${ }_{11}$ Exceptuando Montevideo, apenas se registran guarismos de 6\% o más. 
Los VAB departamentales y sectoriales permiten calcular indicadores de especialización-diversificación productiva.

Inicialmente, se calculan índices de localización (LQ) definidos como:

$$
L Q_{i, j}=\frac{V A B_{j, i} / V A B_{i}}{V A B_{j, U y} / V A B_{U y}}
$$

Para simplificar el análisis se toman promedios del período y se agrupan los sectores en primario (agricultura y ganadería), secundario (minería, industria manufacturera, construcción y electricidad, gas y agua) y terciario (el resto de los sectores) ${ }^{12}$. El índice de localización que aquí se calcula permite comparar, en el año respectivo, la participación de cada sector $j$ en el VAB total del departamento $i$ respecto a la referida participación para el total nacional. Cuando el LQ toma valores por encima de la unidad indica la mayor especialización de la producción en el sector analizado en el departamento, mientras que cuando adopta valores por debajo señala lo contrario. La concentración de la producción en determinados sectores da cuenta de la especialización productiva departamental. Los resultados confirman la fuertísima vocación primaria de la economía uruguaya (figura 1).

En el caso de la producción primaria, solamente tres departamentos presentan valores del indicador por debajo de la unidad: Montevideo, Colonia y Canelones. Justamente, la capital del país, Montevideo, es el único departamento que alcanza, simultáneamente, valores mayores a la unidad en los sectores secundario y terciario. En tanto que Colonia, con un importante desarrollo industrial, comparte con Montevideo la especialización en el sector secundario, y Canelones -asocia- do a una lógica metropolitana- presenta una significativa especialización en el sector terciario. A su vez, la zona sur y litoral (excluyendo Río Negro), aunque con valores menores a la unidad, se diferencia del resto del país al presentar niveles de especialización industrial más elevados.

Algo similar ocurre en el sector terciario, pero con la diferencia de que a este grupo se agregan los departamentos de Rivera y Cerro Largo al norte -en una lógica de frontera- y Florida hacia el centro del país. La evidencia es clara en cuanto a que la localización geográfica de la producción se caracteriza por una muy fuerte vocación primaria porque solamente algunos departamentos ubicados al sur del país, Montevideo, Colonia y Canelones presentan niveles destacados de especialización industrial y de servicios. Esos departamentos son los que lideran el ranking de participación promedio del período en términos de VAB total, por lo que constituyen, entonces, las economías de mayor dimensión económica.

Desde la segunda mitad del siglo xix, Uruguay fue un creciente receptor de inmigrantes europeos (mayoría de españoles, italianos y franceses). En buena medida, la llegada de inmigrantes respondió a la preocupación de quienes veían en el poblamiento de la campaña un factor clave para el desarrollo del país. Sin embargo, su destino principal fue la capital y los departamentos vecinos. El censo general de población de 1860 muestra que más de tres cuartas partes de los inmigrantes europeos se afincaron en Montevideo (54,2\%), Canelones (22,5\%) y Colonia $(4,2 \%)$, mientras que al final del período de investigación, en 1908 , los datos del censo comprueban que la situación no había cambiado sustancialmente. No es casual que los departamentos más receptivos fueran los del área metropolitana porque era allí en dónde el crecimiento de la ciudad coincidía con las mayores oportunidades que brindaban las actividades industriales, comerciales y de servicios (Klaczko y Rial, 1981). A su vez, la actividad agrícola con destino al mercado interno se realizaba en zonas cercanas al principal mercado de consumo, constituido

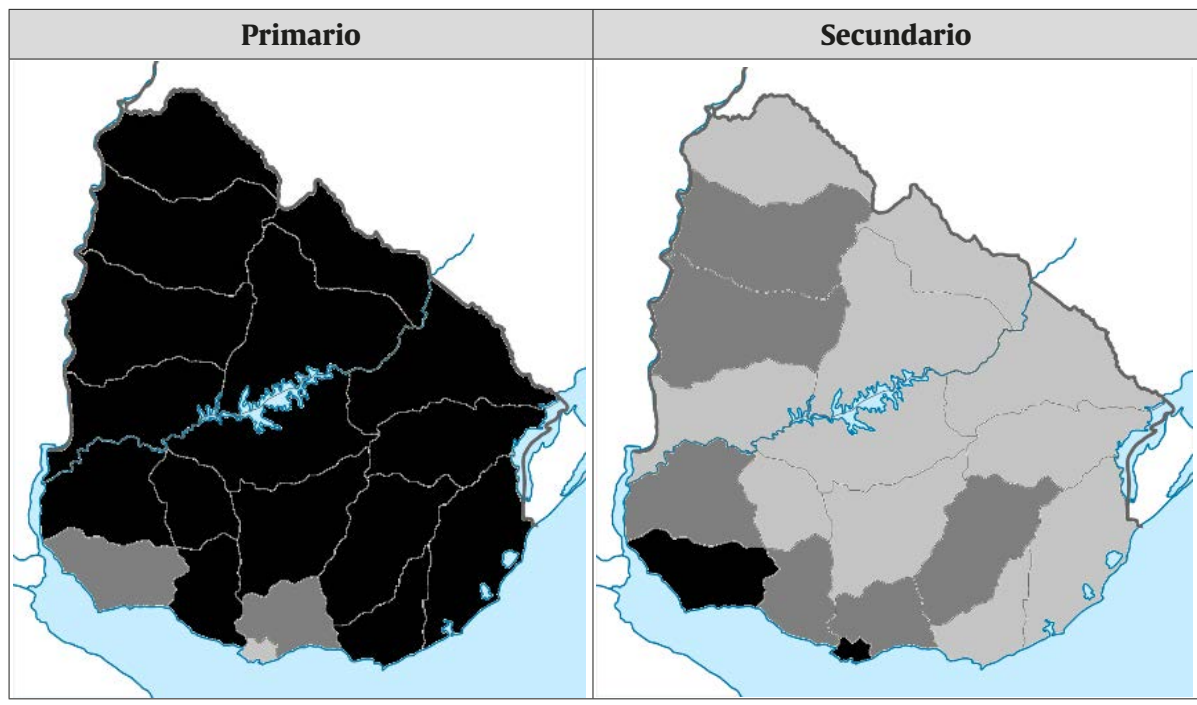

Figura 1. Indicador de localización por agregados económicos (promedios 1872-1908) Fuente: elaboración propia

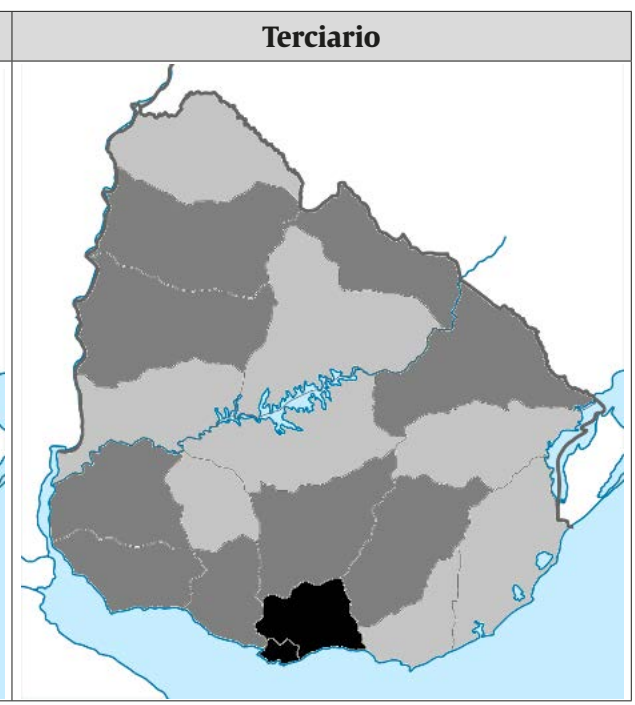

LQ $>1$
LQ entre 0,5 y 1
LQ $<0,5$

12 En Castro Scavone y Willebald (2019) se reportan los resultados anuales de los LQ. 
por Montevideo. El caso de Colonia es paradigmático porque, de los departamentos del interior del país, fue el que recibió mayor cantidad de inmigrantes europeos. Las colonias agrícolas que se instalaron en este departamento, y lo distinguieron en el paisaje agrario nacional, debieron alentar la llegada de población de los países de origen. En los departamentos centro y norte del país, caracterizados por una fuerte especialización pecuaria, la absoluta preponderancia de la ganadería extensiva debió operar como un desincentivo para el arraigo de los inmigrantes.

Si bien la ausencia de información no ha permitido analizar en detalle los movimientos migratorios internos, un aspecto que debe ser tenido en cuenta es el que refiere a la nacionalidad de los inmigrantes, ya que su experiencia, conocimiento y destrezas incorporadas pueden ser un factor relevante para explicar el desempeño económico de las regiones en las cuales se establecieron. La inmigración europea fue las más destacada, y se puede afirmar que fueron en su mayoría italianos y españoles quienes eligieron Uruguay para establecerse. En el censo de 1860 los registros permitieron comprobar que el 78\% de los españoles censados tenían residencia en uno de esos tres departamentos del sur del país, y una cifra levemente mayor (86,2\%) resultó para el caso de los italianos. Como antes, al final del período la situación se mantenía sin cambios drásticos. Los registros del censo de 1908 fueron, para esos tres departamentos en conjunto, de $74,3 \%$ de españoles y de $76,1 \%$ de italianos. Así, a falta de incorporar más información para mejorar el análisis, se puede decir que la zona metropolitana de Montevideo, que a diferencia del resto del país presentó un destacado crecimiento del sector industrial, comercial y de servicios, tuvo en la inmigración (con una fuerte presencia de españoles e italianos y en proporciones similares) un acicate importante para su desarrollo.

Un segundo ejercicio consiste en calcular el índice de especialización de Krugman (KSI) (Krugman, 1991), el cual considera la totalidad de los sectores disponibles en cada estimación. Se define como sigue:

$$
K S I_{i}=\sum_{i=1}^{n}\left|\frac{V A B_{i, j}}{V A B_{i}}-\frac{V A B_{k, j}}{V A B_{k}}\right|
$$

Donde $\mathrm{VAB}_{\mathrm{ij}}$ es el VAB del $i$-ésimo departamento correspondiente al $j$-ésimo sector, con $i=1 . .19 \mathrm{y} j=$ agricultura, ganadería, minería, industria manufacturera, construcción, electricidad, gas y agua, comercio, transporte y almacenamiento, comunicaciones, servicios de intermediación financiera, Administración pública, actividades inmobiliarias, empresariales y de alquiler, y otros servicios.

$\mathrm{VAB}_{\mathrm{j}, \mathrm{k}}$ : igual que el anterior pero para cada uno de los otros $k$ departamentos tomados como referencia.

Tabla 2

Índice de especialización departamental (KSI), 1872-1908

\begin{tabular}{lllllllc} 
Departamento & $\mathbf{1 8 7 2}$ & $\mathbf{1 8 8 4}$ & $\mathbf{1 8 9 0}$ & $\mathbf{1 8 9 5}$ & $\mathbf{1 9 0 0}$ & $\mathbf{1 9 0 8}$ & Promedio 1872-1908 \\
\hline Montevideo & 1,30 & 1,27 & 1,20 & 1,25 & 1,17 & 1,19 & 1,23 \\
\hline Canelones & 1,13 & 1,18 & 1,08 & 1,13 & 1,02 & 1,01 & 1,09 \\
\hline Colonia & 0,73 & 0,90 & 0,97 & 0,93 & 1,04 & 0,88 & 0,91 \\
\hline San.José & 0,50 & 0,62 & 0,61 & 0,70 & 0,71 & 0,65 & 0,63 \\
\hline Lavalleja & 0,59 & 0,64 & 0,62 & 0,51 & 0,58 & 0,55 & 0,58 \\
\hline Rivera & 0,59 & 0,53 & 0,56 & 0,49 & 0,51 & 0,46 & 0,52 \\
\hline Paysandú & 0,57 & 0,53 & 0,47 & 0,44 & 0,48 & 0,48 & 0,49 \\
\hline Maldonado & 0,56 & 0,47 & 0,44 & 0,45 & 0,46 & 0,50 & 0,48 \\
\hline Río Negro & 0,53 & 0,52 & 0,46 & 0,45 & 0,48 & 0,43 & 0,48 \\
\hline Artigas & 0,46 & 0,46 & 0,50 & 0,43 & 0,48 & 0,50 & 0,47 \\
\hline Treinta.y.Tres & 0,45 & 0,47 & 0,47 & 0,46 & 0,44 & 0,47 & 0,46 \\
\hline Florida & 0,45 & 0,45 & 0,46 & 0,46 & 0,47 & 0,45 & 0,46 \\
\hline Tacuarembó & 0,46 & 0,49 & 0,47 & 0,44 & 0,42 & 0,44 & 0,45 \\
\hline Durazno & 0,43 & 0,43 & 0,48 & 0,50 & 0,43 & 0,42 & 0,45 \\
\hline Flores & 0,45 & 0,44 & 0,43 & 0,47 & 0,46 & 0,43 & 0,45 \\
\hline Rocha & 0,50 & 0,45 & 0,42 & 0,43 & 0,44 & 0,43 & 0,45 \\
\hline Cerro.Largo & 0,42 & 0,44 & 0,44 & 0,43 & 0,45 & 0,45 & 0,44 \\
\hline Salto & 0,46 & 0,44 & 0,42 & 0,43 & 0,46 & 0,42 & 0,44 \\
\hline Soriano & 0,42 & 0,43 & 0,41 & 0,41 & 0,41 & 0,43 & 0,42 \\
\hline & & & & & & & \\
\hline
\end{tabular}

Fuente: elaboración propia. 
El indicador varía entre 0 (estructuras productivas idénticas) y 2 (no hay absolutamente ninguna superposición) y mide el grado en que se diferencian las estructuras productivas departamentales. El cálculo se realiza para cada uno de los benchmarks temporales, tomando el promedio de las dieciocho comparaciones a que se somete cada departamento. Los departamentos con valores más elevados del indicador (aquellos que resultan más diferentes al resto) son: Montevideo, Canelones y Colonia, mientras que en un escalón más abajo se encuentran San José y Lavalleja. La diversificación productiva basada en la incipiente producción industrial y de servicios tuvo su epicentro en Montevideo y se reflejó en dos departamentos del sur del país: Colonia para el caso de la industria y Canelones en servicios. El resto de los departamentos -incluidos San José y Lavalleja, con valores de 0,63 y 0,58 respectivamente- presentan registros muy bajos del indicador.

\subsection{VAB per cápita}

Se clasifican los departamentos según el lugar que ocupan en el ranking de VAB per cápita (pc) (tabla 3).
Desde el último tercio del siglo xix, Montevideo se constituyó en el principal centro urbano, con una elite europeizada y un puerto de aguas profundas, líder en el Río de la Plata, y que representa la región más rica del país. Se trata de niveles de PIB pc altos en términos comparados frente, incluso, a otros países. Por ejemplo, en 1895 superaba al promedio de Argentina (cuando, comparando ambos países, el PIB pc de Uruguay representaba solo el 63\% del argentino) y de Gran Bretaña. En tanto que, contrastando regiones, se ubicaba en posiciones muy similares a las de las provincias de Buenos Aires, La Pampa y Entre Ríos (Badía-Miró et al., 2020) ${ }^{13}$.

Podría conjeturarse que durante las últimas décadas del siglo xix la diversificación productiva no habría sido un factor determinante, salvo para el caso de Montevideo. Excluyendo a la capital del país, los departamentos que lideran el ranking (Río Negro, Artigas, Paysandú, Flores) presentan niveles intermedios del indicador KSI y todos ellos son departamentos de alta especialización agropecuaria (solo Paysandú agrega algo de diversificación). Esto es, los departamentos que mejor se acoplaron al modelo agroexportador, en zonas escasamente

\section{Tabla 3}

VAB pc departamental, 1872-1908 (en relación con la media)

\begin{tabular}{|lrrrrrrr|}
\hline Departamento & $\mathbf{1 8 7 2}$ & $\mathbf{1 8 8 4}$ & $\mathbf{1 8 9 0}$ & $\mathbf{1 8 9 5}$ & $\mathbf{1 9 0 0}$ & $\mathbf{1 9 0 8}$ & Promedio 1872-1908 \\
\hline Montevideo & 168 & 162 & 190 & 162 & 154 & 162 & 167 \\
\hline Río.Negro & 168 & 178 & 149 & 166 & 149 & 150 & 160 \\
\hline Artigas & 167 & 132 & 97 & 120 & 106 & 68 & 115 \\
\hline Paysandú & 117 & 124 & 94 & 104 & 108 & 122 & 112 \\
\hline Flores & 103 & 125 & 110 & 119 & 118 & 79 & 109 \\
\hline Colonia & 79 & 100 & 95 & 97 & 137 & 103 & 102 \\
\hline Salto & 90 & 84 & 70 & 84 & 89 & 71 & 81 \\
\hline Rocha & 116 & 80 & 68 & 80 & 79 & 52 & 79 \\
\hline Soriano & 65 & 72 & 63 & 79 & 86 & 107 & 79 \\
\hline Tacuarembó & 111 & 87 & 70 & 77 & 67 & 55 & 78 \\
\hline Rivera & 122 & 78 & 65 & 69 & 58 & 50 & 74 \\
\hline Durazno & 74 & 84 & 67 & 76 & 78 & 53 & 72 \\
\hline Treinta.y.Tres & 74 & 76 & 64 & 76 & 76 & 48 & 69 \\
\hline Maldonado & 73 & 72 & 59 & 74 & 83 & 42 & 67 \\
\hline Cerro.Largo & 63 & 70 & 60 & 70 & 63 & 59 & 64 \\
\hline Florida & 49 & 53 & 47 & 61 & 65 & 84 & 60 \\
\hline San José & 46 & 46 & 40 & 51 & 52 & 91 & 54 \\
\hline Canelones & 41 & 45 & 39 & 49 & 47 & 75 & 49 \\
\hline Lavalleja & 32 & 26 & 24 & 27 & 39 & 38 & 31 \\
\hline Uruguay & $\mathbf{1 0 0}$ & $\mathbf{1 0 0}$ & $\mathbf{1 0 0}$ & $\mathbf{1 0 0}$ & $\mathbf{1 0 0}$ & $\mathbf{1 0 0}$ & $\mathbf{1 0 0}$ \\
\hline
\end{tabular}

Fuente: elaboración propia.

${ }^{13}$ Comparaciones realizadas en Maddison's International Geary-Khamis US dollars (base 2011). 
pobladas, pero con abundantes recursos naturales, fueron los que obtuvieron mayores niveles de ingreso pc.

Estos mismos departamentos -Río Negro, Artigas, Paysandú y Flores-, que se ubican en la parte alta del ranking de VAB pc, pertenecen al litoral y norte del país y son de carácter ganadero, aunque muestran, de todos modos, cierta presencia industrial (a escala del siglo xIX). El caso de mayor notoriedad es el del actual departamento de Río Negro, que estaba contenido en el entonces departamento de Paysandú (hasta el año 1880) y albergaba al único centro industrial del interior realmente destacado, donde se había instalado la empresa internacional Liebig, reconocida mundialmente por su producción de extracto de carne y carne conservada (Lewowicz, 2016).
Unos escalones más abajo se sitúan Colonia y Salto. El primero está ubicado en una zona privilegiada, cercano a la capital del país, con una dotación de tierras de muy buena calidad y disponibilidad de vías marítimas que favorecieron su conectividad. Este departamento también tendrá un protagonismo muy importante en el siglo xx debido a una importante diversificación productiva que ya comienza a dar algunas señales al final de este período. Colonia, al igual que Montevideo y Canelones, presenta una muy baja especialización agropecuaria, pero logra escalar lugares en el ranking por su destacado desarrollo industrial ${ }^{14}$.

El caso de Salto podría asimilarse al de los líderes del período. Se trata de un departamento que combina una fuerte vocación ganadera (al igual que el departamento de Artigas, su

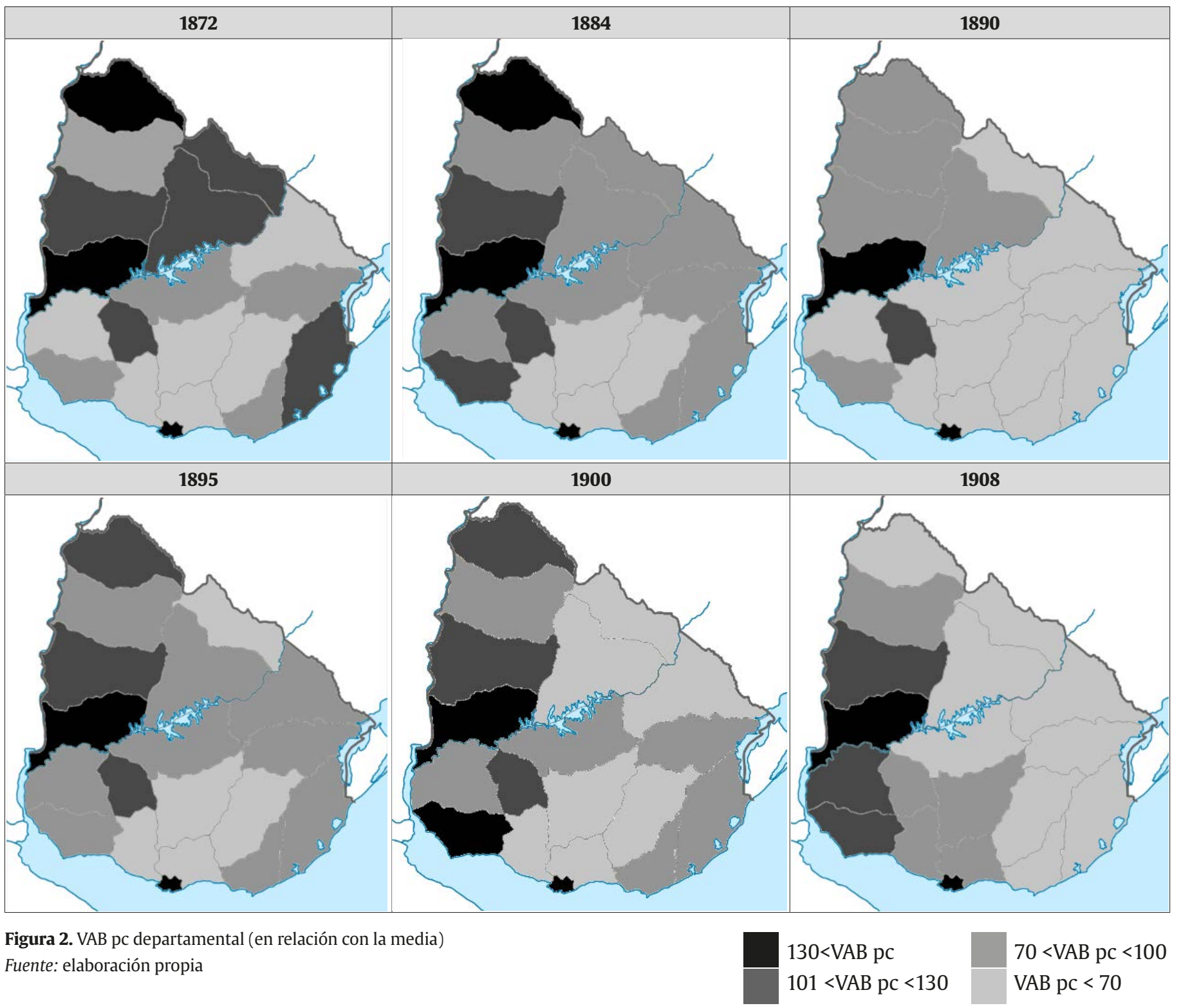

${ }^{14}$ Colonia se ubica en la parte alta del ranking de patentes de giro de la industria manufacturera, y solo es superada por Canelones y Montevideo en el promedio del período. Su estructura industrial daba cuenta de una variada gama de establecimientos: carpinterías, herrerías, destilerías, talleres de reparación, hornos de ladrillos, mataderos y saladeros, zapaterías y talabarterías, sastrerías, confiterías y panaderías. 
vecino al norte) con una incipiente actividad industrial (como es el caso de su vecino al sur, Paysandú).

Bajo una especie de abanico que se abre desde el litoral -la zona más rica del país - se ubican los departamentos de ingreso medio como Rocha, Soriano, Tacuarembó, Rivera, Durazno, Treinta y Tres, Maldonado y Cerro largo, algunos de los cuales son zonas escasamente pobladas y han presentado una fuerte vocación ganadera. A su vez, a la especialización pecuaria podría agregarse una característica compartida por algunos miembros de este grupo, como lo es su posición fronteriza con Brasil. Es probable que el efecto vecindad haya permitido una mayor movilidad de bienes y factores con una incidencia positiva en el ingreso de estos departamentos. Finalmente, el abanico se cierra en el límite de Montevideo, englobando a la zona más pobre del país, compuesta por los departamentos de Florida, San José, Canelones y Lavalleja. Es posible conjeturar que hasta que la zona metropolitana -con Montevideo como centro dinámico - no se consolidó como un mercado ampliado de bienes y factores, suficientemente dinámico como para absorber la creciente oferta de trabajadores expulsados del medio rural, no se afianzó como una región de altos ingresos en el concierto nacional. Con la diversificación productiva impulsada por el crecimiento del sector industrial y de servicios durante el siglo $\mathrm{xx}$, este fenómeno se fue revirtiendo y estos departamentos lograron escalar lugares en el ranking del VAB pc, en un proceso que comenzó a manifestarse al final del período de estudio ${ }^{15}$. En 1908 se puede observar la conformación de una región en forma de L que integra a los departamentos de mayor ingreso pc, en la cual los departamentos del área metropolitana mejoraron sustancialmente su posición en el ranking (figura 2), aunque nunca llegarán a ser líderes y durante varios períodos mantendrán una brecha de desarrollo muy importante respecto de la capital.

Desde diversos flancos se ha discutido la ocupación del espacio y la creación de paisajes agrarios en Uruguay en perspectiva histórica. En particular, haciendo referencia al período 1750-1850 se ha insistido en la idea de complementar las nociones más arraigadas en la historiografía local $-i$. e., que la ocupación del espacio habría ocurrido desde el sur (sobre el océano Atlántico) hacia el norte- con la constatación de la conformación de un paisaje norte-misionero con características propias y dinámicas específicas (Moraes, 2008).

Esto es, ha crecido la convicción de que, efectivamente, el avance de las relaciones económicas sobre el territorio desde Montevideo (desde el sur hacia el norte) habría convivido con la existencia de un dinámico mercado interior de bienes y también de factores, lo que habla de una activa economía agraria no atlántica en el (hoy) norte del país y conectada con las antiguas Misiones Orientales. La evidencia que se presenta en este artículo no hace más que abonar esta idea $-\mathrm{y}$ quizás darle mayor continuidad temporal-al hallar un par de núcleos de alto ingreso en el territorio en la década de 1870; esto es, Montevideo al sur y el territorio al norte del río Negro. Luego de la década de 1890, esta estructura se desdibujará para dar lugar a otra en la cual permanece Montevideo, pero gana preeminencia el litoral sur, sobre la frontera con Argentina.

\footnotetext{
${ }^{15}$ De todos modos, las particularidades del proceso mostrarán un siglo xx en el cual Canelones nunca logrará dejar los lugares finales del ranking en términos de VAB pc (Martínez-Galarraga et al., 2020).
}

\subsection{Desigualdad regional}

Los fuertes diferenciales en términos de VAB pc y la alta concentración de la actividad económica en algunos departamentos redundaron en indicadores de desigualdad relativamente altos en la comparación intertemporal (tabla 4). Índices de Gini en torno a 0,29 (1872-1890) no volverán a repetirse durante todo el siglo $\mathrm{xx}$, y ello es consistente con la fortaleza económica de un Montevideo que, sin embargo, demorará en transformarse en el corazón de la centralidad política ${ }^{16}$.

La evolución del índice de Gini es representada en el gráfico $1^{17}$. Es particularmente significativo el aumento y la caída del indicador antes y luego de 1890. Este año coincide con una de las principales crisis económicas del período analizado y la dimensión financiera de la crisis (aspecto resaltado en la literatura) afectó fundamentalmente a la actividad económica de Montevideo (Nahum, 1998).

\section{Tabla 4}

Desigualdad del VAB pc departamental, 1872-1908

\begin{tabular}{|c|c|c|c|c|c|c|}
\hline $\begin{array}{l}\text { Medidas } \\
\text { de desigualdad }\end{array}$ & 1872 & 1884 & 1890 & 1895 & 1900 & 1908 \\
\hline $\begin{array}{l}\text { Desviación estándar } \\
\text { relativa }\end{array}$ & 0,22 & 0,21 & 0,27 & 0,20 & 0,19 & 0,19 \\
\hline $\begin{array}{l}\text { Coeficiente de } \\
\text { variación }\end{array}$ & 0,51 & 0,48 & 0,62 & 0,47 & 0,44 & 0,44 \\
\hline Desviación estándar & 0,55 & 0,54 & 0,64 & 0,51 & 0,47 & 0,46 \\
\hline Coeficiente de Gini & 0,28 & 0,26 & 0,33 & 0,25 & 0,24 & 0,24 \\
\hline Medida Mehran & 0,41 & 0,39 & 0,46 & 0,37 & 0,36 & 0,35 \\
\hline Medida de Piesch & 0,21 & 0,20 & 0,26 & 0,19 & 0,18 & 0,18 \\
\hline Medida Kakwani & 0,07 & 0,07 & 0,10 & 0,06 & 0,05 & 0,05 \\
\hline $\begin{array}{l}\text { Índice de Theil } \\
\text { (GE (a), a = 1) }\end{array}$ & 0,13 & 0,11 & 0,18 & 0,10 & 0,09 & 0,09 \\
\hline $\operatorname{MLD}(\operatorname{GE}(a), a=0)$ & 0,14 & 0,13 & 0,19 & 0,11 & 0,10 & 0,10 \\
\hline $\begin{array}{l}\text { Índice de entropía } \\
(\mathrm{GE}(\mathrm{a}), \mathrm{a}=-1)\end{array}$ & 0,16 & 0,16 & 0,23 & 0,14 & 0,11 & 0,11 \\
\hline $\begin{array}{l}\text { Coef. de variación } \\
\text { al cuadrado } \\
\text { (GE (a), a = 2) }\end{array}$ & 0,12 & 0,11 & 0,18 & 0,10 & 0,09 & 0,09 \\
\hline p90/p10 & 4,14 & 3,62 & 4,86 & 3,28 & 3,29 & 3,31 \\
\hline p90/p50 & 2,13 & 1,92 & 2,71 & 2,04 & 1,80 & 1,75 \\
\hline
\end{tabular}

Fuente: elaboración propia.

${ }^{16}$ Durante todo ese último tercio del siglo xIx la confrontación política campo/ciudad (interior/Montevideo) fue la constante, con repetidos levantamientos armados y revoluciones. No será hasta 1904-1905 cuando, política y militarmente, Montevideo alcance un predominio decidido y sostenido en el tiempo.

${ }^{17}$ Los otros indicadores de desigualdad siguen trayectorias similares. 


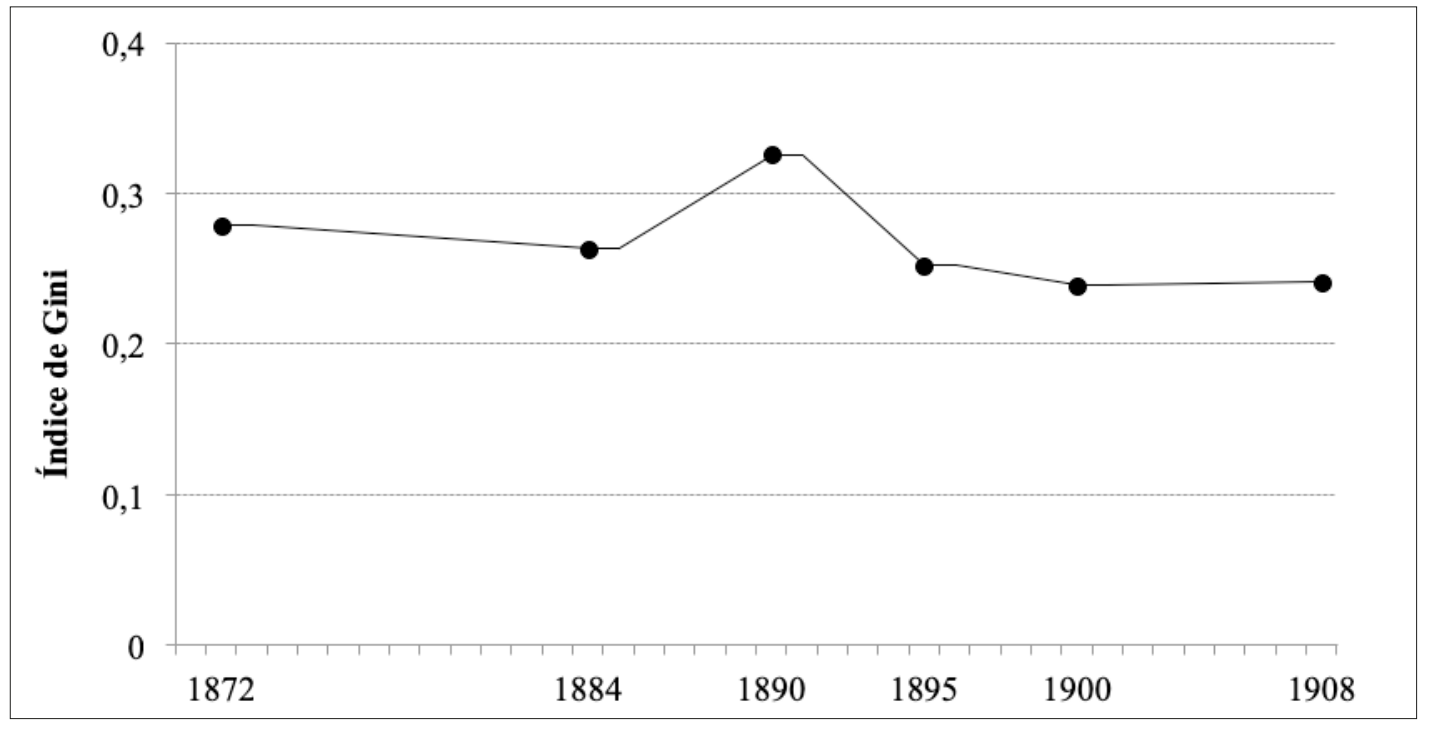

Gráfico 1. Desigualdad regional del ingreso, 1872-1908 Fuente: elaboración propia.
Podría argumentarse que la concentración del ingreso regional en los años previos a la crisis respondió a una creciente actividad especulativa, fundamentalmente urbana, y especialmente montevideana (Buzzetti, 1969), que encontró sus límites al desatarse la crisis de $1890^{18}$. El impacto de la crisis tuvo efectos igualadores producto de haber afectado proporcionalmente más a las zonas urbanas en las cuales las actividades especulativas tuvieron su mayor desarrollo y, especialmente, en la capital del país ${ }^{19}$.

Mirando el momento de boom y depresión, la Crisis de 1890 no hizo más que corregir una experiencia concentradora del ingreso regional que respondía a razones especulativas antes que a fundamentos de la localización productiva. Al menos hasta finales de siglo, estos fundamentos daban cuenta de una evolución firme de mercados regionales que se estaban integrando -donde el ferrocarril habría tenido un rol clave (Díaz, 2014; Barrán y Nahum, 1978) - y varios departamentos ganaban en diversificación productiva, ingresos y fortalecimiento de sus mercados.

Sin embargo, esta tendencia insinúa tener un quiebre -0 ralentización- en el cambio de siglo. Montevideo ganó participación en el período, con un avance industrial relativamente dinámico - la actividad de los frigoríficos será una clara expresión de ello luego de 1911 (Finch, 2005)-y donde los servicios -liderados por un Estado con firmes aspiraciones de expansión-comenzaron una lenta consolidación que no se detendrá en las décadas siguientes.

Previamente se había argumentado que la dotación de tierra para la producción agropecuaria se distribuye con relativa homogeneidad en todo el territorio (ver el cálculo del indica-

\footnotetext{
${ }^{18}$ De hecho, el registro más alto de VAB pc de Montevideo ocurre en 1890 (tabla 3).

19 Para comprobar estas apreciaciones se realizó un ejercicio estándar de contribución al crecimiento por sectores de actividad para Montevideo (ver anexo tabla A.1). En los albores de la crisis de 1890 hubo un crecimiento de la contribución al crecimiento de los sectores vinculados al comercio y, fundamentalmente, a los servicios. A su vez, en los años posteriores a la crisis (que se recogen en el año 1895) se observa que la mayor caída de la contribución al crecimiento se produjo en esos sectores.
}

dor LQ en la sección 4) y que, por lo tanto, habría operado como un factor de desconcentración del ingreso nacional en el período. Para captar el efecto de la especialización primaria en la dinámica de la desigualdad se realizaron dos ejercicios de carácter contrafactual. El primer ejercicio se basó en suponer que la producción primaria registrada en 1872 se mantuvo constante a lo largo del período, mientras que en el segundo ejercicio se asumió la ausencia de producción primaria en los departamentos. Para evaluar el resultado del primer ejercicio se contrastó el índice de Gini efectivo con el contrafactual. Se verificaron valores muy similares del indicador, aunque mayores en el caso del índice calculado a partir del ejercicio contrafactual. De este modo, se obtuvo evidencia de que si la producción primaria se hubiera mantenido constante, la desigualdad hubiera sido mayor (anexo gráfico A.1). Los resultados del segundo ejercicio (bajo el supuesto de ausencia de actividad primaria) dan cuenta de una desigualdad sensiblemente mayor en el escenario contrafactual (anexo cuadro A.2).

Una de las expectativas de este trabajo era aproximarse a resultados previos obtenidos en Martínez-Galarraga et al. (2019) para 1908. En este se siguió la metodología propuesta originalmente por Geary y Stark (2001) para el cálculo de VAB regionales basada en datos de empleo y salarios por sector de actividad. Si bien, como era de esperar, existen diferencias, las comparaciones son alentadoras y permiten validar la metodología utilizada en este trabajo ${ }^{20}$.

\subsection{Convergencia regional}

Con el objetivo de complementar y enriquecer el análisis de la desigualdad regional, se realizaron ejercicios estándar de convergencia propuestos originalmente por Barro y Sala-i-Martín (1990). En línea con estudios recientes que han utilizado este tipo de técnicas para estudiar la convergencia condicional entre regiones, considerando el nivel inicial de

\footnotetext{
${ }^{20}$ Niveles de desigualdad regional similares y ranking de VAB pc próximos y altamente correlacionados.
} 
renta y algunas características específicas de las economías (Badia-Miró et. al., 2018), se propone incluir dummies en la estimación para recoger efectos adicionales.

El estudio de la $\beta$-convergencia absoluta supondría, implícitamente, que los fundamentos económicos de los departamentos no diferirían significativamente a lo largo del tiempo. Este supuesto es sumamente restrictivo y altamente improbable, por lo que se optó por estudiar la $\beta$-convergencia condicionada que se correspondería a diferentes estructuras económicas y de dotación de recursos entre departamentos.

Aprovechando la variabilidad espacial y temporal de los datos, se construye un panel que combina las observaciones de sección cruzada (diecinueve departamentos) con la serie temporal (seis referencias temporales que cubren el período 1872-1908). La propuesta metodológica se basa en utilizar estos datos para estimar un modelo de efectos fijos. El uso de un modelo de efectos fijos asegura que se tendrán en cuenta los factores invariantes en el tiempo y específicos para cada región en la ecuación de convergencia.

Partiendo de la ecuación 3, la estimación del coeficiente $\beta$, además de capturar la correlación parcial entre la tasa de crecimiento y el nivel del VAB pc, brinda una medida de la velocidad de convergencia de cada región a su respectivo steady-state.

$$
\dot{y}_{i t}=\alpha+\mu_{i}+\delta_{t}+\beta \ln y_{i t-1}+\varepsilon_{i t}
$$

Donde $\dot{y}$ es la tasa de crecimiento promedio anual del VAB pc (a precios constantes del año 2005) ${ }^{21}$ en el subperíodo $t$ (con $\mathrm{t}=1872-1884,1884-1890,1890-1895,1895-1900,1900-1908)$ para cada departamento $i, y_{\mathrm{it}-1}$ es el nivel del VAB pc rezagado un período en cada región (esto es, al inicio del período en cuestión), ${ }^{22} \alpha$ es la constante del modelo, $\mu_{\mathrm{i}}$ son los efectos fijos, $\delta_{\mathrm{t}}$ son los efectos temporales y $\varepsilon_{\mathrm{it}}$ una perturbación aleatoria con media cero y varianza $\sigma_{\varepsilon}^{2}$.

Considerando dos modelos - sin incluir dummies temporales (modelo I) e incluyéndolas para 1884, 1890, 1895 y 1900 (modelo II) - se obtuvo, para todo el período de análisis (18721908 ), evidencia de convergencia tipo $\beta$ (condicionada en ambos casos), confirmada por el signo negativo de la estimación puntual (tabla 5). Si bien el ajuste de la estimación mejora cuando se incluyen dummies temporales (el $\mathrm{R}^{2}$ overall es 0,1245 en el modelo I y 0,3025 en el modelo II), el mismo no es suficientemente bueno, por lo cual se presume que la especificación del modelo deja fuera variables explicativas relevantes.

Finalmente, en la estimación de panel el coeficiente $\beta$ resulta negativo y significativo al $1 \%$ (el resultado es $\beta=-0,9649$ ). La velocidad de convergencia, indicativa del ritmo al cual las economías se acercan a su estado estacionario, es $1,2 \%$ anual ${ }^{23}$. El valor obtenido, si bien es bajo, no está alejado del valor de referencia habitualmente considerado en la literatura ( $2 \%$; Sala-i-Martin, 1996) ${ }^{24}$.

\footnotetext{
${ }^{21}$ Ante la inexistencia de series de precios por departamento, se utiliza un deflactor a nivel nacional, suponiendo homogeneidad de precios por regiones (deflactor de Román y Willebald, 2019).

22 Esto es, en el primer subperíodo se considera la tasa de crecimiento anual de 1872-1884 como variable explicada y el nivel del VAB pc en 1872 como explicativa.

${ }^{23}$ Calculada como: $v=\log (1-\beta T) / T$ (Sala-i-Martin, 1996).

${ }^{24} \mathrm{Si}$ el cálculo se realiza con la estimación del modelo II $(\beta=-0,6502)$ el resultado es $0,74 \%$.
}

\section{Tabla 5}

Regresión de convergencia (variable dependiente: tasa de crecimiento del VAB: $\dot{y}$ )

\begin{tabular}{lcc|}
\hline Variables & Modelo I (EF) & Modelo II (EF) \\
\hline log(vab) $)_{\text {-1 }}$ & $-0.9649^{* * *}$ & $-0,6502^{* * *}$ \\
& $(0,000)$ & $(0,000)$ \\
d (1884) & & $-0,0652$ \\
& & $(0,209)$ \\
\hline d (1890) & & $-0,2531^{* * *}$ \\
\hline d (1895) & & $(0,000)$ \\
\hline d (1900) & & $-0,0097$ \\
& & $(0,772)$ \\
Constante & $9,48^{* * *}$ & $-0,1947$ \\
\hline Within & $(0,000)$ & $(0,000)$ \\
Between & 0,5244 & $6,501^{* * *}$ \\
\hline Overall & 0,2409 & $(0,000)$ \\
\hline N & 0,1245 & 0,7656 \\
\hline
\end{tabular}

Todos los coeficientes fueron estimados con errores estándar robustos: $\left({ }^{*}\right)$ : significativo al 10\%; $\left(^{* *}\right)$ : significativo al $5 \%$; $\left.{ }^{* * *}\right)$ : significativo al $1 \%$.

Las variables dummies d (1872) y d (1908) fueron omitidas por colinealidad.

\section{Consideraciones finales}

El análisis presentado permite obtener dos conclusiones principales. En primer lugar, es muy significativo el alto peso que históricamente tuvo Montevideo en la generación local de valor agregado. Es indudable que su carácter portuario, su temprana identificación como centro administrativo y de gobierno nacional y su permanente caracterización como centro atractor (de bienes y factores) y dinámico mercado ha llevado a colocarlo a la cabeza en la estructura de la producción de Uruguay. Se trata de un rasgo que se extenderá durante todo el siglo xx y las primeras décadas del xxi y pautará un desarrollo regional en Uruguay sostenidamente desbalanceado.

En segundo lugar, se constató evidencia de convergencia tipo $\beta$ condicional, lo cual no hizo más que confirmar la tendencia decreciente de la desigualdad encontrada para el período, confirmando las hipótesis del trabajo. En el caso de Uruguay no era esperable asociar la Primera Globalización con el primer tramo de la forma de U invertida (Williamson, 1965), sino que, por el contrario, otras fuerzas fueron las que prevalecieron. En particular, la ausencia de cambio estructural (Román y Willebald, 2019) habría dado lugar a una acción más intensa de las fuerzas centrífugas, producto probable- 
mente de la materialización en los mercados internacionales de una riqueza -la ganadera - que era posible realizar en los diversos puntos de la república y podía colocarse a relativo bajo costo en puertos de ultramar. La conectividad creciente que fue brindando el ferrocarril desde los tempranos años de la década de 1870 se agregó -aunque a veces también compitió - con la que proporcionaban desde mucho antes los ríos -sobre todo el río Uruguay - y que encontraba como puerta de salida de la producción nacional no solo al puerto de Montevideo, sino también al de Buenos Aires. En contraste con la fuerte presencia de la producción agropecuaria en todo el territorio nacional, la actividad industrial y de servicios tendió a concentrarse en el sur y fue en los departamentos que alcanzaron mayores niveles de diversificación de sus estructuras productivas en donde la actividad industrial (Montevideo y Colonia) y de servicios (Montevideo y Canelones) tendió a localizarse.

De todos modos, la evolución igualadora no estuvo exenta de reversiones. Tanto en la década de 1880 como en la primera del siglo xx, las fuerzas centrípetas habrían tenido una mayor influencia, lo que aumentó los niveles de desigualdad. En el primer caso, a la creciente importancia de Montevideo como centro urbano y administrativo, mercado de bienes y servicios y centro dinámico del mercado de bienes y factores, se le agregó un período de fuerte dinamismo especulativo en la capital del país, con expresiones comerciales e inmobiliarias muy claras que significaron, al menos en el corto plazo, creación de actividades económicas y generación aumentada de ingresos. La crisis económica y financiera de 1890 afectó a la actividad de comercio y servicios (en especial financieros o de carácter especulativo) que se desarrollaron, fundamentalmente, en Montevideo, y por esa vía pudo frenarse el incipiente proceso concentrador de la capital, recortando las rentas financieras y sosteniendo a la economía sobre la base del dinamismo de las actividades más tradicionales.

En cambio, a comienzos del siglo xx vuelven a manifestarse señales de una acción creciente de las fuerzas centrípetas que, al contario de la década de 1880 , podrían estar más asociadas con una incipiente industria manufacturera, proceso que la literatura local identifica con la «temprana industrialización» (Bértola, 2000; Jacob, 1988), caracterizada por la expansión de talleres y pequeñas fábricas junto con el establecimiento de grandes frigoríficos en la capital.

El liderazgo de la capital, Montevideo, prácticamente se constató durante todo el período. Esta persistencia contrasta con la transformación ocurrida en los restantes departamentos. Hacia la década de 1870 el segundo núcleo de departamentos ricos se verificaba al norte del río Negro, bajo consignas de territorios de baja población, abundantes recursos naturales y relaciones dinámicas con los países vecinos (el sur de Brasil y el litoral argentino). En cambio, luego de casi cuatro décadas de evolución, hacia 1908 parece conformarse una zona relativamente más rica, que adopta una forma de L e incluye a los departamentos del sur y litoral del país, siendo este un rasgo estructural de la distribución regional de la producción de Uruguay en el largo plazo.

\section{Agradecimientos}

Agradecemos el apoyo financiero del Programa I+D de CSIC-UDELAR (Comisión Sectorial de Investigación Científica-Universidad de la República) 2012. También queremos agra- decer especialmente a Julio Martínez-Galarraga, Adrián Rodríguez Miranda, Sebastián Goinheix y Sabrina Siniscalchi sus colaboraciones en trabajos anteriores que fueron valiosos aportes para esta investigación, así como a Camilo Martínez y Maximiliano Presa por compartir sus datos con nosotros. Igualmente, agradecemos los comentarios y sugerencias recibidos en varios seminarios y conferencias (VI Jornadas Académicas de la Facultad de Ciencias Económicas y de Administración, Montevideo, octubre de 2016; XXXI Jornadas Anuales de Economía, Banco Central del Uruguay, Montevideo, agosto de 2016; Workshop de Jóvenes Investigadores en Historia Económica y Demografía Histórica de los siglos xviII y xix, Montevideo, junio de 2016; V Congreso Latinoamericano de Historia Económica, Universidade de São Paulo, Brasil, julio de 2016). Como es de uso, somos responsables de todos los errores que aún permanezcan.

\section{Fuentes}

Aguiar, J. J. (1901). Censo ganadero de la República Oriental del Uruguay, 1900. Imprenta Rural.

Censos Nacionales del Uruguay, varios años $(1860,1908)$.

Contaduría General de la Nación, varios años $(1893,1902)$.

Presupuesto General de Gastos, Uruguay.

Dirección General de Estadística y Censos, varios años (1860, 1884, 1887, 1889, 1890, 1892, 1894, 1895, 1898, 1900 у 1908). Anuario Estadístico de la República Oriental del Uruguay. Montevideo: Imprenta Artística y Encuadernación de Dornaleche y Reyes.

Legislativo, P., varios años (1884, 1890, 1895, 1900 y 1908). Registro Nacional de Leyes y Decretos.

\section{Bibliografía}

Acevedo, E. (1933). Anales de la Universidad, tomo II. Montevideo: Casa A. Barreiro y Ramos.

Acevedo, E. (1936). Anales de la Universidad. Montevideo: Casa A. Barreiro y Ramos.

Alonso Pérez, M. y Risso, W. (2012). Una aproximación al PBI turístico departamental de Uruguay 2010. Montevideo.

Badia-Miró, M., Guilera, J. y Lains, P. (2012). «Regional incomes in Portugal: industrialization, integration and inequality, 1890-1980», Revista de Historia Económica/Journal of Iberian and Latin American Economic History, 30 (2), 225-244.

Badia-Miró, M., Nicolini, E. y Willebald, H. (2020). «Spatial inequality in Latin America (1895-2010): Convergence and clusters in a longrun approach", en Badía-Miró, M., Tirado-Fabregat, D. y Willebald, H. (eds.). Time and Space: Latin American Regional Development in Historical Perspective. London: Palgrave Macmillan.

Badia-Miró, M., Nicolini, E. y Willebald, H. (2018). «Growth and regional disparities in South America, 1890-1960», Journal of Interdisciplinary History, 49 (1), 117-139.

Balbis, J. (2005). «La evolución del precio de la tierra en Uruguay (19141924)», Quantum, 2 (5), 114-141.

Baldwin, R. E., Forslid, R., Martin, Ph., Ottaviano, G. I. P. y Robert-Nicoud, F. (2003). Economic Geography and Public Policy. Princeton, NJ: Princeton University Press.

Baracchini, H. (1981). Historia de las comunicaciones en el Uruguay. Montevideo: Universidad de la República.

Barrán, J. P. y Nahum, B. (1978). Historia rural del Uruguay moderno: tomo 7. Agricultura, crédito y transporte bajo Batlle (1905-1914). Montevideo: Banda Oriental.

Sala-i-Martin, X. (2000). Apuntes de crecimiento económico. Antoni Bosch Editor.

Baumann, N. (2017). Historia de la minería en el Uruguay a comienzo del s. xx (1903-1930). Una historia poco conocida. Montevideo: Roger Jolly Casa Editorial. 
Bertino, M., Bertoni, R., Tajam, H. y Yaffé, J. (2005). La economía del primer batllismo y los años veinte. Historia Económica del Uruguay, tomo 3. Montevideo: Universidad de la República (Uruguay), Facultad de Ciencias Económicas y de Administración, Instituto de Economía.

Bértola, L. (2000). "Crecimiento de la industria temprana en Uruguay», en Bértola L. (ed.). Ensayos de Historia Económica. Uruguay y la región en la economía mundial. 1870-1990. Ediciones Trilce, pp. 149-166.

Bértola, L. et al. (1998). El PIB Uruguayo1870-1936 y otras estimaciones. Montevideo: Programa de Historia Económica, Facultad de Ciencias Sociales.

Bertoni, R. (2002). Economía y cambio técnico: adopción y difusión de la energía eléctrica en Uruguay 1880-1980. Maestría en Historia Económica, Facultad de Ciencias Sociales-UDELAR, Uruguay.

BROU (Banco de la República Oriental del Uruguay) (1965). Cuentas Nacionales. Montevideo: Departamento de Investigaciones Económicas.

Buyst, E. (2011). "Continuity and change in regional disparities in Belgium during the twentieth century", Journal of Historical Geography, 37 (3), 329-337.

Buzzetti, J. (1969). Historia económica y financiera del Uruguay. Montevideo: Imprenta La Paz.

Caselli, F. y Coleman, W. J. (2001). «Cross-Country Technology Diffusion: The Case of Computers", American Economic Review, 91(2), 328-335.

Castro Scavone, P. y Willebald, H. (2019). Producto regional en Uruguay durante la Primera Globalización (1872-1908): desigualad decreciente y convergencia entre regiones. Serie Documentos de Trabajo, DT 25/2019. Universidad de la República, Uruguay: Instituto de Economía, Facultad de Ciencias Económicas y Administración.

Crafts, N. (2005). "Regional GDP in Britain, 1871-1911: some estimates», Scottish Journal of Political Economy, 52 (1), 54-64.

Díaz Steinberg, G. M. (2014). La inversión ferroviaria en el Uruguay antes de 1914: rentabilidad privada, subsidios e impacto económico [máster]. Universidad de la República, Montevideo.

Enflo, K., Henning, M. y Schön, L. (2014). «Swedish regional GDP 18552000: estimations and general trends in the Swedish regional system", Research in Economic History, 30, 47-89.

Felice, E. (2011). «Regional value added in Italy, 1891-2001, and the foundation of a long-term picture», Economic History Review, 64 (3), 929-950.

Finch, H. (2005). La economía política del Uruguay contemporáneo: 1870-2000, Montevideo: Ediciones de la Banda Oriental.

Fujita, M., Krugman, P. y Venables, A. (2001). The spatial economy: Cities, regions, and international trade. MIT Press.

Geary, F. y Stark, T. (2002). «Examining Ireland's post-famine economic growth performance», The Economic Journal, 112, 919-935.

Jacob, R. (1988). "La industrialización anterior a 1930: crónica de un debate nunca realizado", Industria Uruguaya.

Klaczko, Jaime y Rial, Juan (1981). Uruguay, el país urbano. Montevideo: CLACSO, Comisión de Desarrollo Urbano y Regional, Ediciones de la Banda Oriental.

Krugman, P. (1991). «Increasing returns and economic geography», Journal of Political Economy, 99 (3), 483-499.

Leamer, E. (1995). The Heckscher-Ohlin model in theory and practice. Princeton: Princeton University.

Lezama, G. y Willebald, H. (2019). «Inequality in pre-income survey times: a methodological proposal», Review of Income and Wealth, 66 (4), 931-957.

Lewowicz, L. (2016). LEMCO: un coloso de la industria cárnica en Fray Bentos, Uruguay. Montevideo: Zona Editorial.

Martínez-Galarraga, J., Rodríguez-Miranda, A. y Willebald, H. (2020). «Patterns of regional income distribution in Uruguay (1872-2012): a story of agglomeration, natural resources and public policies», en Badia-Miró, M., Tirado-Fabregat, D. y Willebald, H. (eds.). Time and Space -Latin American Regional Development in Historical Perspective, London: Palgrave.

Martínez-Galarraga, J., Rodríguez Miranda, A., Siniscalchi, S. y Willebald, H. (2019). La distribución regional de la producción en Uruguay, 19081975: propuesta metodológica y fuente. Serie Documento de Trabajo, DT 13/2019. Uruguay: Instituto de Economía, Facultad de Ciencias Económicas y de Administración, Universidad de la República.
Martínez Rodríguez, C. (2019). La construcción del Estado Oriental del Uruguay (c. 1853-1893). Otra mirada con foco en la evolución histórica de sus funciones [máster]. Universidad de la República, Montevideo.

Millot, J. y Bertino, M. (1996). Historia económica del Uruguay, tomo II: 1860-1910. Montevideo: Instituto de Economía-Facultad de Ciencias Económicas y de la Administración, Universidad de la República-Fondo de Cultura Universitaria.

Molinari, L. y Prieto, S. (2011). Estimación de las cuentas regionales y sus limitaciones. El caso uruguayo. Documento de Trabajo del BCU, 122011. Montevideo.

Moraes, I. (2008). La pradera perdida. Historia y economía del agro uruguayo: una visión de largo plazo, 1760-1970. Montevideo: Linardi y Risso.

Nahum, B. (1998). La crisis de 1890, tomo 1, La correspondencia de Alberto Nin. Ediciones de la Banda Oriental.

Nathan M. (2014). Empalme de las estimaciones y proyecciones de población anual por sexo y edad de INE-CELADE Rev. 1998 e INE Rev. 2013.

Oficina de Planeamiento y Presupuesto (OPP) (2005a). Departamentalización del producto bruto interno de Uruguay. Periodo: 1985-2003. Oficina de Planeamiento y Presupuesto, Unidad de Desarrollo Municipal.

Oficina de Planeamiento y Presupuesto (OPP) (2005b). Análisis de la regionalización para el desarrollo local en Uruguay. Oficina de Planeamiento y Presupuesto, Unidad de Desarrollo Municipal; AECID.

Pellegrino, A., (s. f.). Un ensayo de estimación de la población de Uruguay 1885-1962. Documento de trabajo inédito. Programa de Población, Facultad de Ciencias Sociales, UDELAR.

Peralta, F. (2008). Los intendentes desde la historia, en este presente y hacia el futuro, a 100 años de la Ley de creación de la figura de intendente municipal. Montevideo: Oficina de Planeamiento y Presupuesto, Programa Uruguay Integra.

Rial, J. (1983). Población y desarrollo de un pequeño país. Uruguay, 1930. Montevideo: Acali.

Rodríguez Miranda, A. (2010a). Desarrollo económico en el noreste de Uruguay: una aproximación a partir de la organización productiva y la articulación rural urbana [tesis]. Universidad Autónoma de Madrid.

Rodríguez Miranda, A. (2010b). Desarrollo económico en el noreste de Uruguay: articulación rural-urbana y organización productiva. DT 3/10. Montevideo: Instituto de Economía, FCEA-UDELAR.

Rodríguez Miranda, A. y Goinheix, S. (2018). Estimación del VAB departamental en Uruguay y evolución en el período 1981-2011. Serie Documentos de Trabajo; 3/18. Uruguay: Instituto de Economía, Facultad de Ciencias Económicas y de Administración, Universidad de la República.

Román, C. y Willebald, H. (2019). Structural change in a small natural resource intensive economy. Switching between diversification and reprimarization. Serie Documentos de Trabajo, DT 31/2019. Uruguay: Instituto de Economía, Facultad de Ciencias Económicas y de Administración, Universidad de la República.

Rosés, J., Martinez-Galarraga J. y Tirado D. A. (2010). «The upswing of regional income inequality in Spain (1860-1930)», Explorations in Economic History, 47 (2), 244-257.

Samuelson, P. A. (1949). «International factor-price equalisation once again", The Economic Journal, 59 (234), 181-197.

UDELAR (Universidad de la República). Facultad de Ciencias Económicas y de Administración (FCEyA) (1995). La Economía Uruguaya en los Noventa. Análisis y perspectiva de largo plazo. Convenio UTE-Universidad de la República. Montevideo: Artes Gráficas.

UDELAR (Universidad de la República). Facultad de Ciencias Económicas y de Administración (FCEyA) (1998). La economía uruguaya en los noventa. Convenio UTE-Universidad de la República. Montevideo: Artes Gráficas.

Vaillant, A. (1873). La República Oriental del Uruguay en la Exposición de Viena. Montevideo: Imprimerie de La Tribuna.

Williamson, J. G. (1965). «Regional inequality and the process of national development: a description of the patterns», Economic Development and Cultural Change, 13 (4, Part 2), 1-84.

Willebald, H. (2015). «Distributive patterns in settler economies: agrarian income inequality during the First Globalization (1870-1913)», Historia Agraria. Revista de Agricultura e Historia Rural, 66, 75-104. 
Anexo

Tabla A.1.

Contribución sectorial al crecimiento económico en Montevideo 1872-1908 (\%)

\begin{tabular}{|c|c|c|c|c|c|c|}
\hline Actividades & 1872 & 1884 & 1890 & 1895 & 1900 & 1908 \\
\hline Agrícola & - & $-0,7$ & $-0,2$ & 0,1 & 0,1 & 0,6 \\
\hline Pecuario & - & 0,0 & 0,0 & 0,0 & 0,3 & 0,2 \\
\hline Minería & - & 0,2 & 0,1 & $-0,1$ & 0,1 & 0,2 \\
\hline Industria Manufacturera & - & 8,6 & 6,8 & 0,0 & 0,7 & 20,1 \\
\hline Construcción & - & 10,8 & 4,5 & $-6,2$ & 1,8 & 1,1 \\
\hline EGA & - & $-0,6$ & 1,3 & $-0,1$ & 1,8 & 0,9 \\
\hline Comercio & - & 29,4 & 6,9 & $-6,9$ & $-0,5$ & 32,8 \\
\hline Transporte y almacenamiento & - & 5,2 & 2,9 & 0,8 & 0,8 & 6,3 \\
\hline Comunicaciones & - & 0,1 & 0,5 & 0,1 & 0,3 & 0,2 \\
\hline Servicios de intermediación Financiera & - & $-5,5$ & 12,8 & $-3,5$ & 5,8 & $-3,2$ \\
\hline Servicios & - & 0,1 & 0,7 & $-0,2$ & 0,0 & 0,9 \\
\hline Administración Pública & - & 4,4 & 5,5 & $-1,9$ & 2,6 & 7,4 \\
\hline $\begin{array}{l}\text { Actividades inmobiliarias, empresariales } \\
\text { y de alquiler }\end{array}$ & - & 4,7 & 1,4 & $-0,4$ & 1,2 & 2,4 \\
\hline VAB total & - & 56,8 & 43,1 & $-18,2$ & 15,0 & 69,8 \\
\hline
\end{tabular}

\section{Gráfico A.1}

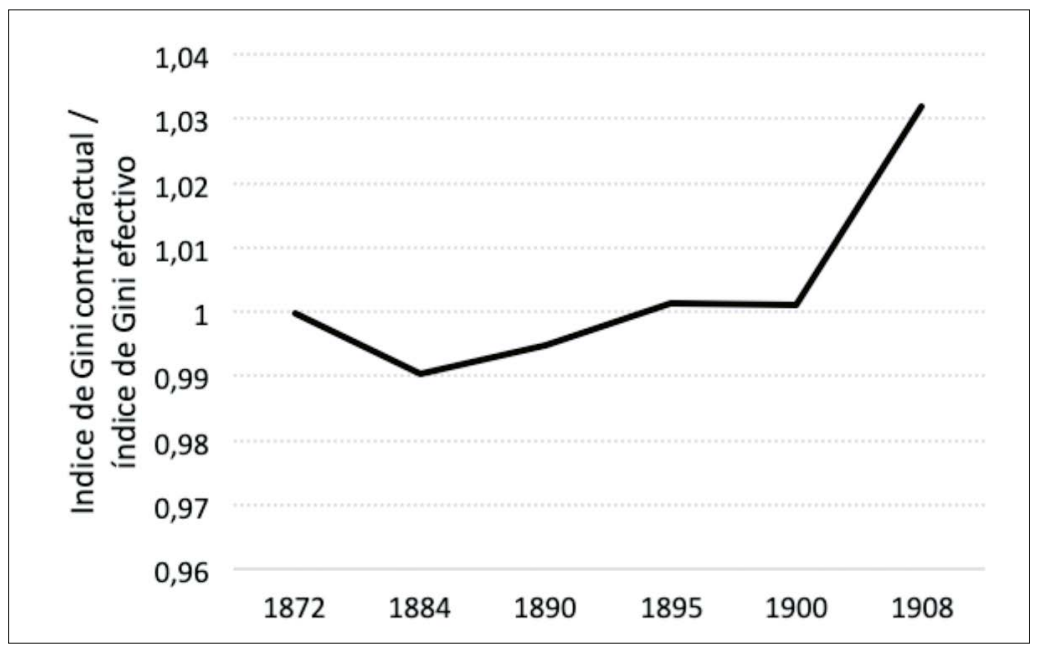

Nota: el índice de Gini contrafactual se calculó asumiendo que la actividad primaria departamental de 1872 se mantuvo constante a lo largo del período.

Tabla A.2.

\begin{tabular}{|c|c|c|c|}
\hline \multirow{2}{*}{ Año } & \multicolumn{2}{|c|}{ Índice de Gini } & \multirow{2}{*}{$\begin{array}{l}\text { Contrafactual / } \\
\text { Efectivo }\end{array}$} \\
\hline & Efectivo & Contrafactual & \\
\hline 1872 & 0,278 & 0,468 & 1,688 \\
\hline 1884 & 0,262 & 0,446 & 1,702 \\
\hline 1890 & 0,325 & 0,487 & 1,498 \\
\hline 1895 & 0,251 & 0,444 & 1,769 \\
\hline 1900 & 0,238 & 0,407 & 1,711 \\
\hline 1908 & 0,240 & 0,427 & 1,777 \\
\hline
\end{tabular}

Nota: el índice de Gini contrafactual se calculó asumiendo la ausencia de actividad primaria a lo largo del período. 